\title{
Design of Energy Scavengers of Structural Health Monitoring Systems Using Genetically Optimized Neural Network Systems
}

\author{
Ibrahim N. Tansel, Mustafa Demetgull, ${ }^{1,}$, \\ Rene A. Leon, Aylin Yenilmez ${ }^{2}$ and Ahmed Yapici \\ Mechanical Engineering Department, Florida International University \\ 10555 West Flagler Street, Miami, FL 33174, USA \\ ${ }^{1}$ Mechanical Department, Technical Education Faculty, \\ Marmara University, Goztepe, Istanbul, Turkey \\ ${ }^{2}$ Istanbul Technical University, Faculty of Mechanical Engineering Gumussuyu, Istanbul, Turkey
}

(Received September 23, 2008; accepted February 12, 2009)

Key words: optimization, neural network, genetic algorithm, GONNS, piezoelectric

Energy scavengers are a promising alternative for powering the thousands of sensors of next-generation air vehicles. Genetically Optimized Neural Network Systems (GONNS) is proposed as the first step for the optimization of energy scavengers by considering the ambient vibration, available space, and allowable weight. GONNS conveniently represents the complex systems with multiple artificial neural networks (ANNs) and are used to determine optional operating conditions using one or more genetic algorithms (GAs). Single- and multiple-cluster modes of the GONNS were used in the study to match the dynamic characteristics of the energy scavenger to the ambient vibrations and to fit the system into the available space. The single-cluster mode represented the relationship between the inputs (frequency, beam length, and mass) and two outputs (voltage and displacement amplitudes) with separate ANNs and optimized the system using a single GA. Six ANNs and three GAs working in three groups optimized the system in the multiple-cluster mode of the GONNS.

\section{Introduction}

Future aerospace vehicles will use elaborate integrated systems health management (ISHM) tools to achieve the targeted safety, low-cost maintenance, operation readiness, and quick repair goals. Instead of wiring all the sensors, on-the-spot energy generation methods such as solar cells, thermoelectric units, and energy scavengers ${ }^{(1)}$ may be used to simplify the infrastructure and reduce the weight. Energy scavengers are an excellent alternative power source particularly at the hard-to-reach places of the vehicle. The dimensions and the mass of energy scavengers should be selected carefully to match their ${ }^{*}$ Corresponding author: e-mail: mdemetgul@marmara.edu.tr 
resonance frequency with the ambient vibration and to fit them into the available space. Tuning a large number of energy scavengers for their location will be a demanding task. In this paper, use of Genetically Optimized Neural Network Systems (GONNS) is proposed to determine the initial dimensions of the energy scavengers.

Piezoelectric materials generate electricity when their dimensions are changed by applying tension or compression. The generated electricity is proportional to the strain and, of course, to the load, which creates the stress.(2) The piezoelectric effects have been studied for a long time and these materials have been used to build sensors and actuators. ${ }^{(3-7)}$ Most of the piezoelectric materials are affordable and may be adapted to many applications with minimal design changes. The efficiency to transform mechanical energy to electrical energy depends on the type of material. The piezoelectric materials such as lead zirconate titanate (PZT), quick pack (QP), monolithic piezoceramic materials embedded in an epoxy matrix, and microfiber composite (MFC) manufactured out of piezofibers have different characteristics. ${ }^{(8-11)}$

Backpropagation (BP) ${ }^{(12,13)}$ is one of the most popular ANNs. It may be used for classification and mapping applications. GAs have been widely used for optimization. ${ }^{(14)}$ Instead of using the analytical models, simulated annealing, fuzzy logic, and ANNs have been used with the GAs. ${ }^{(15)}$ The GA is generally slow; however, it finds the optimal solutions without converging to local minima. In this study, GONNS was selected to avoid the development of neither the analytical models, which will require many approximations because of the complex design of the piezoelectric strip actuators, nor fitting empirical models. GONNS uses multiple ANNs to model the system and determine the optimal values using GA. ${ }^{(16)}$

Depending on the complexity of the problem, GONNS uses one or more clusters. Each cluster generally has multiple ANNs and one GA. In this study, both modes of the GONNS were used.

The theoretical background of the components of GONNS will be introduced briefly in the next section. Implementation of the GONNS for the design of energy scavengers of the aerospace vehicles, experimental setup, results, and conclusions will follow.

\section{Theoretical Background}

Many implementations of the $\mathrm{BP}^{(12-13)}$ are available. In addition to the number of inputs and outputs, the user determines the number of hidden layers and their nodes. Most of the programmers connect each node to all the nodes of the next layer. The nodes of the hidden and output layers use simple transfer functions to process their inputs. These transfer functions multiply the incoming values by weight and use a simple function to determine their output. The sigmoid is the most commonly used function. Linear, Gaussian, and various hyperbolic functions have also been used depending on the need. The network starts to process the incoming training signals with arbitrary weights. The error is calculated by comparing the output of the network with the corresponding values in the training file. All the weights are adjusted by backpropagating the errors through the network at each interaction. This process is repeated many times until the network's output errors are reduced to an acceptable level. The user selects the learning rate and the momentum to control the speed and stability of the network. 
GAs are developed to imitate the biological evolution principles. Natural selection and survival of the fittest are the most commonly used approaches. ${ }^{(17,18)}$ The user determines the number of binary digits to be assigned for each parameter and their boundaries. Additional bits can be assigned for switches. All the parameters and the switches are represented with a chromosome. The algorithm tries to find the best 0 and 1 combination of this string either to minimize or to maximize the objective function. The penalty functions might be used to force some of the parameters to stay in the selected range. The user generally selects the population size, the number of children for each set of parents, and the probability of mutation. The chromosomes are generated randomly for the first generation. Generally, GAs follow a five-step optimization procedure that includes: (1) selection of the mating parents, (2) selection of the hereditary chromosomes from the parents, (3) gene crossover, (4) gene mutation, and (5) creation of the next generation.

The GONNS uses the ANNs for modeling and GA(s) for optimization. Generally, an ANN is trained to represent the relationship between all the inputs and one of the outputs of the system. For most of the systems, more than one output is considered and multiple ANNs are used. Our GONNS application aims to minimize or maximize one of the outputs while the other outputs are maintained at the desired ranges. GONNS may be operated either in the single- or multiple-cluster modes. In the single-cluster mode, multiple ANNs and one GA are used. In the multiple-cluster modes, ANN and GA groups are used. All the clusters are optimized at the same time.

\section{The Problem and the Proposed Procedure}

The most common design of the energy scavengers is presented in Fig. 1. A mass is attached to one side of a piezoelectric element. The piezoelectric element is fixed from the opposite end and a cantilever beam is created. The dimensions of the beam and mass should be carefully selected to fit the system into the available space, not to crack the piezoelectric element during the flight, to avoid striking the cover, and to match the resonance frequency with the dominant frequency of the ambient vibrations as much as possible. Since the piezoelectric element has a complex multilayer structure, the tiny

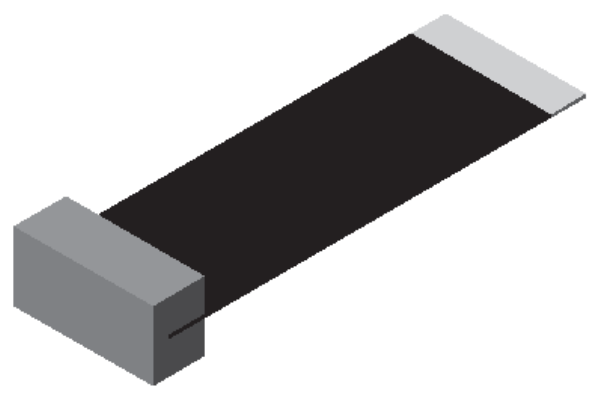

Fig. 1. Piezoelectric element and mass. 
support, which holds the beam, flexes, and the mass cannot be applied to a single spot; thus, the development of an analytical model is difficult. Finite element method (FEM) may be used; however, estimation of the dynamic responses and voltage takes a very long time. GONNS is proposed to represent the experimental results in a very compact form using ANNs.

In this study, both modes of the GONNS were used. In the first mode, the GONNS used 2 ANNs and one GA. Two ANNs were trained to represent the relationship between three inputs and one of the two considered outputs. The inputs were the frequency, length of the piezoelectric stripe actuator, and the mass. The single outputs of two neural networks were the amplitudes of the voltage and the tip displacements. The diagram of this mode is presented in Fig. 2.

In the second mode, separate clusters were assigned to the masses tested in the experiments (Fig. 3). Generally, the multi-cluster mode is more accurate. Six ANNs were used with two inputs and one output. The inputs were the frequency and length of the beam. One of the ANNs estimates the amplitude of the generated voltage while the other estimates the amplitude of the beam tip oscillation. Each cluster was assigned to make the optimization for a single mass value that they were trained for.

\section{Experimental Setup}

American Piezo Ceramics, Inc. (APCI) (Catalog No. 40-1010 (600/200/0.60-SA)) piezoelectric stripe actuators were used in the experiments. Their dimensions were 53 $\mathrm{mm}$ by $20 \mathrm{~mm}$ with the thickness of $0.6 \mathrm{~mm}$. The diagram of the experimental setup is presented in Fig. 4(a). The beam was attached to a V6100-6 VT5 Vibration Test System. A harmonic signal was generated using a BK Precision 4017 signal generator connected to the MacroTech Crown XTI 1000 power amplifier of the exciter. A Kaman Displacement Measuring System KD 2310-2S was used to measure the amplitudes of the vibrations of the shaker and the beam tip. The signal of the function generator, the output of the piezoelectric actuator, and the proximity sensor were connected to a Nicolet Integra 10 digital oscilloscope. The experimental setup is shown in Fig. 4(b).

The experimental data was collected with three different lead weights attached to the beam tip with $1.9,4.7$, and $6.85 \mathrm{~g}$ masses. The lengths of the beam were set to 21 ,

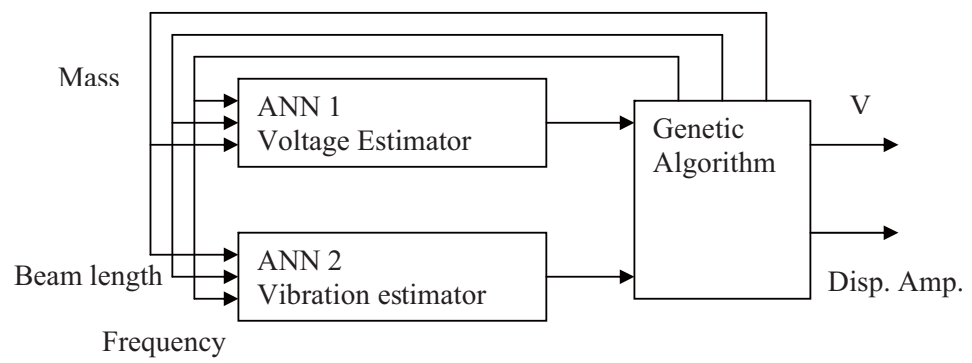

Fig. 2. Optimization of the operating conditions using the GONNS at the single-cluster mode. 


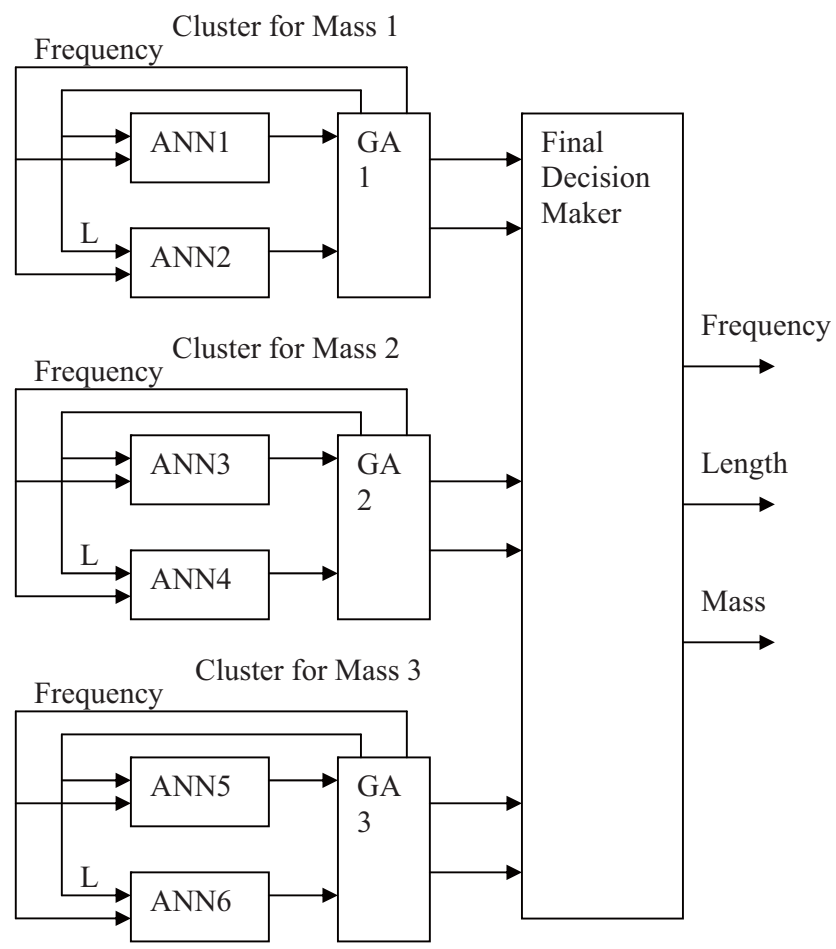

Fig. 3. Optimization of the battery dimensions in the multiple-cluster mode.

32, and $43 \mathrm{~mm}$ in the experiments. Each experiment was repeated with $10 \mathrm{~Hz}$ intervals between 10 and $190 \mathrm{~Hz}$. One more experiment was carried out for each beam length and mass combination to find the resonance frequency and the maximum amplitudes of the generated voltage and tool tip displacements. The experimental data was collected using the Integra 10 digital oscilloscope. The data was stored on floppy disks. The experimental data was assessed using Microsoft Excel and peak-to-peak voltage was found for each experiment. The amplitude of the displacement was found using the calibration coefficient of the Kaman Displacement Measuring System data.

\section{Results and Discussion}

The modeling of the dynamic characteristics of the piezoelectric beams using the ANNs was a great challenge compared with many other applications. Frequency response curves make sharp peaks around the natural frequency of the beam. It was not easy to orient these sharp peaks to ANNs with two or three inputs. Some of the ANNs made large errors at the frequencies surrounding the peaks. These errors were not detected during the training process since there was no training data at those frequencies. 
(a)

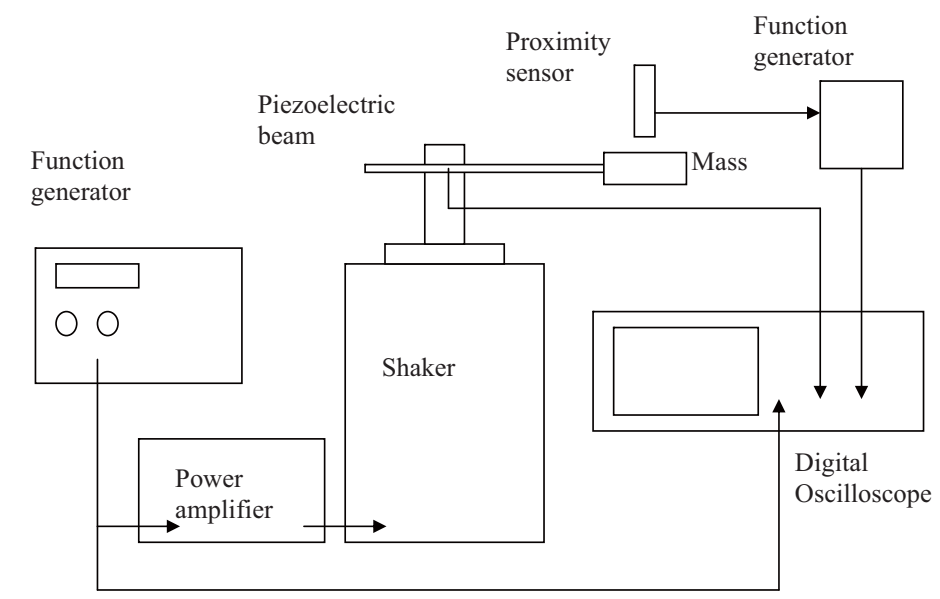

(b)

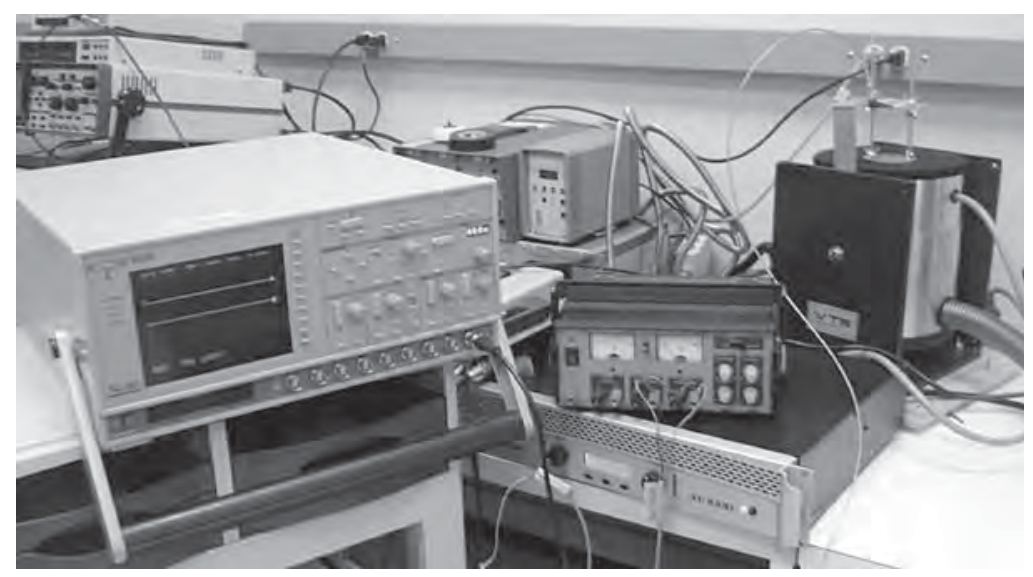

Fig. 4. (a) Diagram of the experimental setup. (b) Picture of the experimental setup.

Two modes of the GONNS were tested in this study because the number of inputs of the ANNs of the multiple-cluster modes is one less than the other mode, and ANNs learn the training data better. In the first mode, two neural networks with three inputs were trained and the optimum was found using one GA. The second mode used three clusters with two ANNs and one GA in each cluster. Each cluster was optimized for one of the tested masses.

In the first mode (Fig. 2), two ANNs were trained using 177 experimentally obtained cases. ANNs made over one million iterations. The average estimation errors of the ANNs are presented in Table 1. The genetic algorithm used the ANNs with 16 and 10 hidden nodes for the estimation of the amplitude of the generated voltage and tool tip displacement, respectively. 
Table 1

Average estimation error of the 2 ANNs (in the first mode) after over 1 million iterations. Results are presented for 10, 16, and 20 hidden nodes.

\begin{tabular}{ccc}
\hline Number of hidden nodes & $\begin{array}{c}\text { Voltage estimating with ANN } \\
\text { (Average estimation error with } \\
\text { respect to the full range) }\end{array}$ & $\begin{array}{c}\text { Amplitude estimating with ANN } \\
\text { (Average estimation error with } \\
\text { respect to the full range) }\end{array}$ \\
\hline 10 & 1.93 & 1.31 \\
16 & 1.73 & 1.37 \\
20 & 1.87 & 1.83 \\
\hline
\end{tabular}

In the second mode (Fig. 3), 6 ANNs were trained to estimate the amplitudes of either the generated voltage or the tool tip vibration for three different mass values. The average estimation errors of the ANNs (Table 2) were lower when 8 hidden nodes were used; however, there were some large errors at the surroundings of the peaks at the natural frequencies. We preferred to use the ANNs with 12 hidden nodes for the optimization using the GA.

The accuracy of the trained ANNs is presented with the 3-D graphs in Figs. 6 and 7. The experimentally observed amplitudes of the generated voltage and tool tip displacements are presented in Fig. 5. The parameters of experiments are presented in Table 3. In these 3-D graphs, the locations of the icons indicate the experimental conditions including the frequency, and the beam length and mass as per Table 3. The match of the experimental values in Fig. 5, estimations of the 2 ANNs of the singlecluster GONNS in Fig. 6, and estimations of the 6 ANNs of the multiple-cluster GONNS in Fig. 7 indicate the excellent performance of the ANNs.

The limitations of even excellent models can be seen in Fig. 5 of ref. 19. Two stacks of 2 piezowafers with $45.974 \times 20.574 \times 0.254 \mathrm{~mm}^{3}$ dimensions attached to a plate were used. There was no additional mass. The analytical model estimated the three modes extremely well. However, at low frequencies up to half of the natural frequency, the estimation of the analytical model significantly deviated from the experimental results. The experiments were performed using harmonic function in this study to obtain the best possible accuracy. Also, the ANNs performed well at this critical low frequency range as shown in Figs. 5-7.

The genetic algorithm quickly converged and estimated the best operating condition. For one case, the selection of the parameters for the optimization and the results are presented in Figs. 8 and 9. The user wanted to maximize the voltage variation while the amplitude of the beam tip displacement was kept below $4 \mathrm{~mm}$ (Fig. 8) when the scavenger operates at a location with the ambient vibration of $80 \mathrm{~Hz}$ dominant frequency. The optimization of the genetic algorithm was stopped after $30 \mathrm{~s}$ (Fig.9). Over 2400 iterations were completed in that time interval. The GONNS recommended a $21 \mathrm{~mm}$ beam length and $2.39 \mathrm{~g}$ mass. 
Table 2

Average estimation error of the 6 ANNs (in the second mode) after over 1 million iterations. Results are presented for 8 and 12 hidden nodes.

\begin{tabular}{crcc}
\hline Mass (g) & $\begin{array}{c}\text { Number of hidden nodes } \\
\text { Voltage estimating with ANN } \\
\text { (Average estimation error } \\
\text { with respect to the full range) }\end{array}$ & $\begin{array}{l}\text { Amplitude estimating with ANN } \\
\text { (Average estimation error with } \\
\text { respect to the full range) }\end{array}$ \\
\hline 1.9 & 8 & 1.117 & 0.53 \\
1.9 & 12 & 1.29 & 1.62 \\
4.7 & 8 & 1.22 & 0.63 \\
4.7 & 12 & 0.82 & 0.92 \\
6.85 & 8 & 0.65 & 0.496 \\
6.85 & 12 & 1.15 & 0.87 \\
\hline
\end{tabular}

Table 3

Parameters of experiments.

\begin{tabular}{ccc}
\hline Experiments & Beam length $(\mathrm{mm})$ & Mass $(\mathrm{g})$ \\
\hline 1 & 21 & 1.9 \\
2 & 21 & 4.7 \\
3 & 21 & 6.85 \\
4 & 32 & 1.9 \\
5 & 32 & 4.7 \\
6 & 32 & 6.85 \\
7 & 43 & 1.9 \\
8 & 43 & 4.7 \\
9 & 43 & 6.85 \\
\hline
\end{tabular}

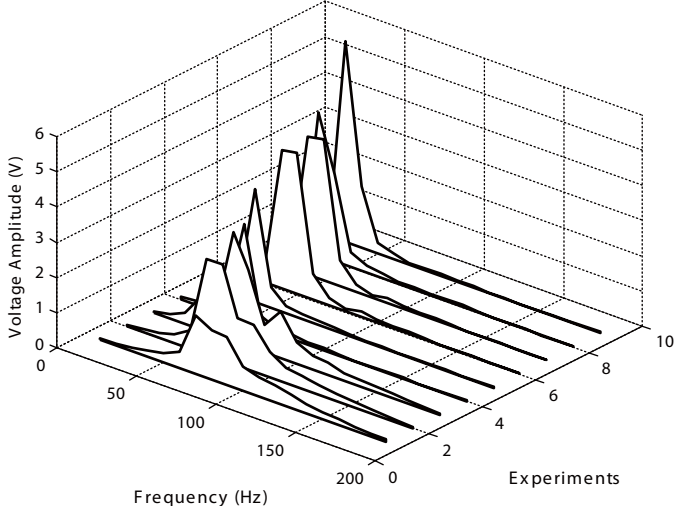

(a)

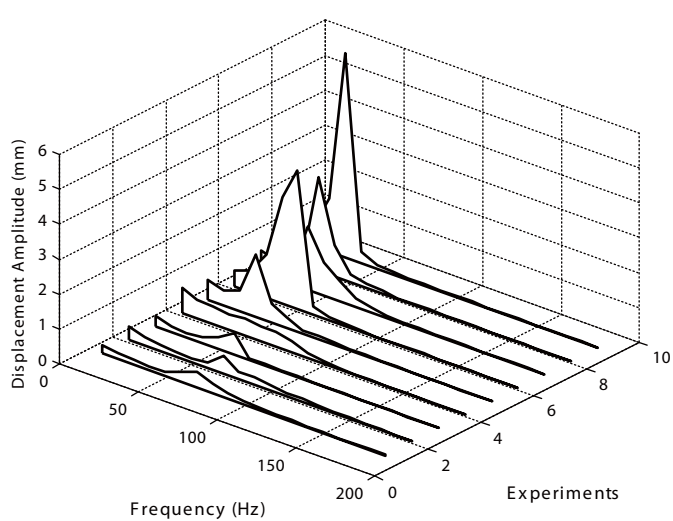

(b)

Fig. 5. 3-D graph of the experimental results. The amplitude of the generated voltage (a) and the tool tip vibration (b) are presented. 


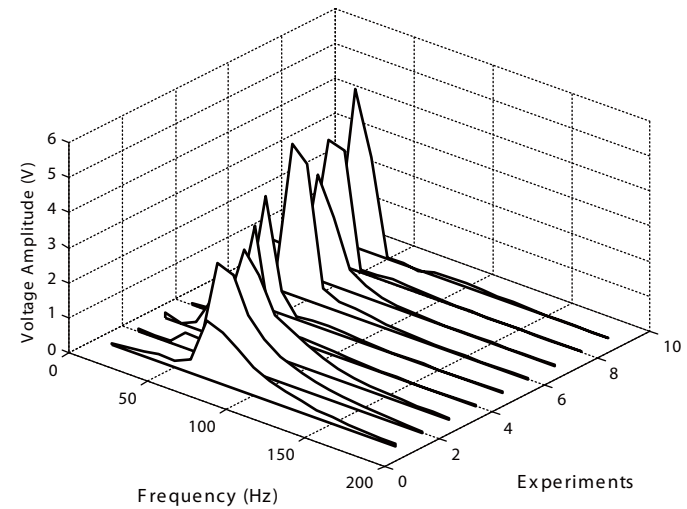

(a)

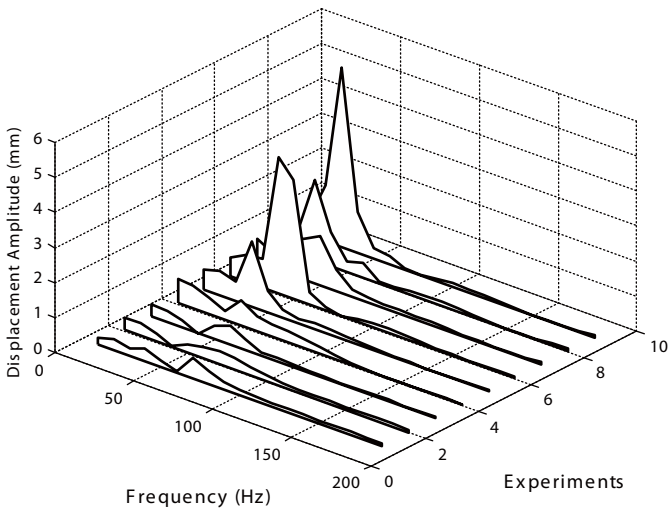

(b)

Fig. 6. 3-D graph of the estimations of two ANNs of the single-cluster GONNS in Fig. 2. The similarities of the data of the amplitudes of the generated voltage (a) and the tool tip vibration (b) to those shown in Figs. 5(a) and 5(b) indicate the excellent performance of the ANNs.

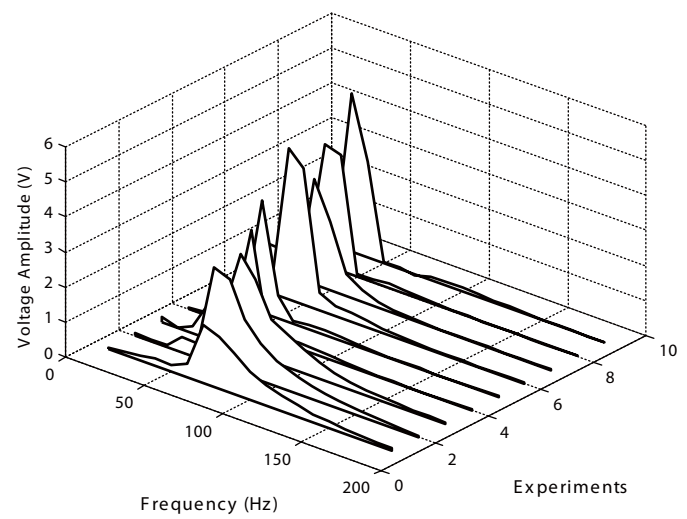

(a)

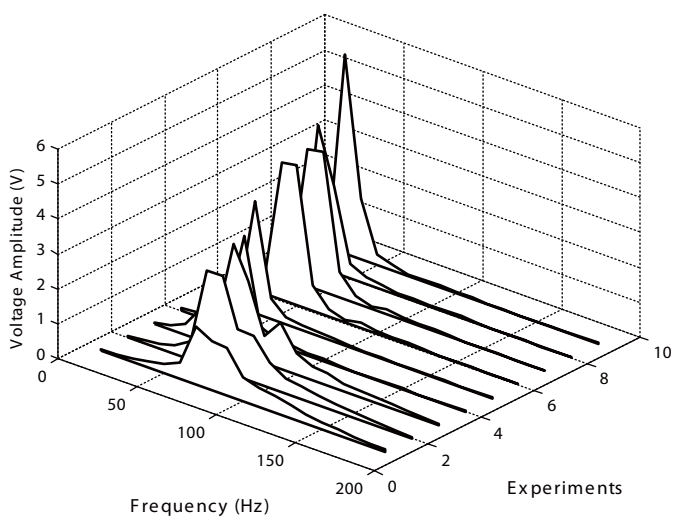

(b)

Fig. 7. 3-D graph of the estimation of six ANNs of the multiple-cluster mode of GONNS in Fig. 3. The similarities of the data of the amplitudes of the generated voltage (a) and the tool tip vibration (b) to those shown in the Figs. 5(a) and 5(b) indicate the excellent performance of the ANNs. 


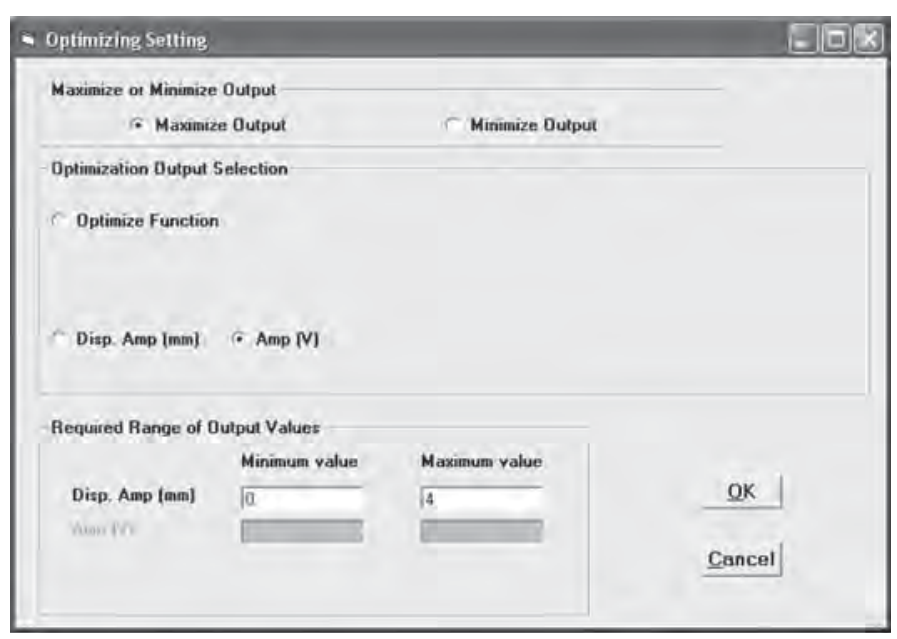

Fig. 8. Selection of optimization goals using the GONNS.

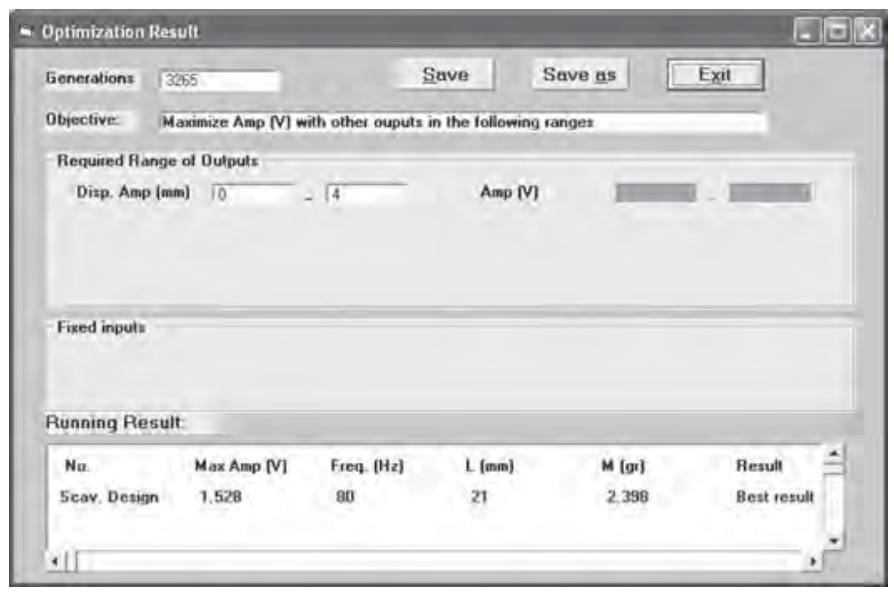

Fig. 9. Optimization results with the GONNS.

The GONNS, which use multiple-clusters, also recommended the similar dimensions and mass weight when the optimization was requested under similar conditions (Fig. 10). In this mode, GONNS can recommend only one of the masses used for the collection of the training data. When we allowed the GONNS to use the full range and asked to find the optimum operating conditions, it selected the longest beam with the heaviest mass (Fig. 11).

Generally, the ambient vibration frequency and available space will be given. The engineers should select the range of the inputs and one of the outputs while the other output is maximized or minimized. The frequency range that will be selected is very small in most of the applications just around the dominant frequency of the ambient vibration. The length of the beam and most probably the allowable tip vibration 


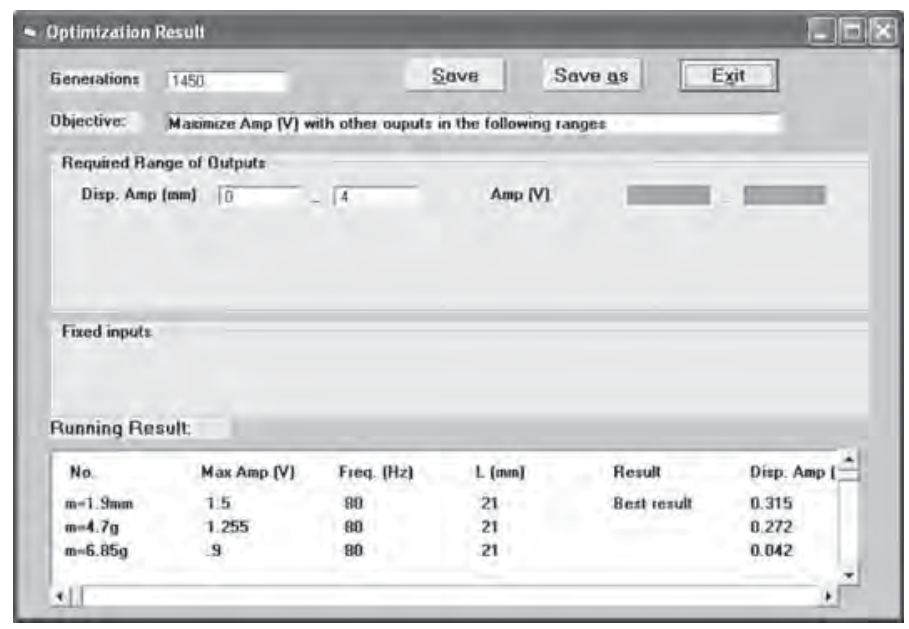

Fig. 10. For the installation of the scavenger on a machine part with $80 \mathrm{~Hz}$ natural frequency, GONNS recommended the shortest beam length and the smallest mass to operate very close to the natural frequency of the system.

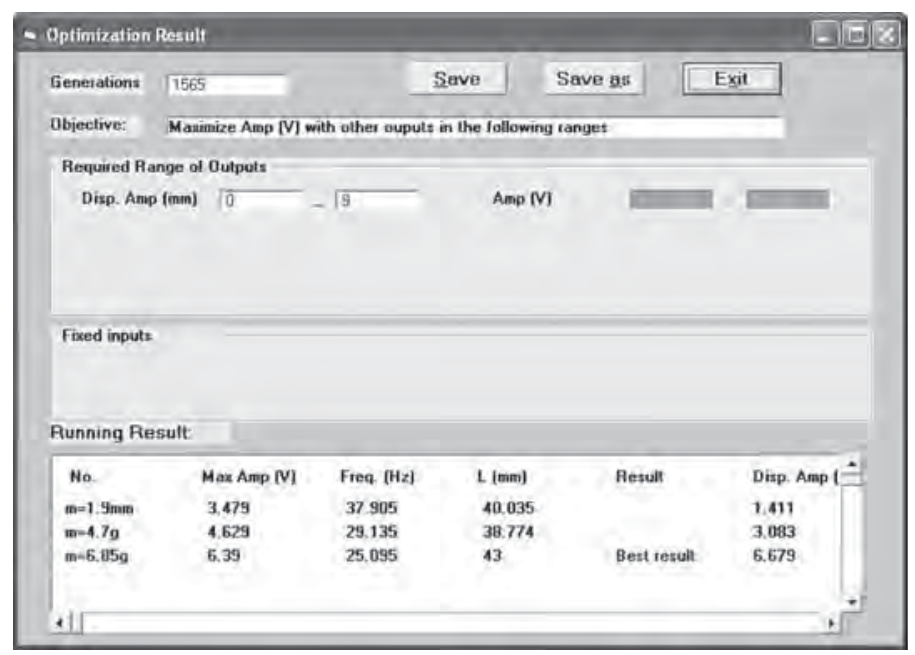

Fig. 11. The GONNS suggested using the maximum piezoelectric element length and heaviest mass when we allowed it to use the experimental data collection range.

amplitude will be selected to fit the system into the desired space. The optimization will be performed to obtain the best possible amplitude for the generated voltage. The user may also determine the range of the amplitude of the generated voltage by considering his/her circuit and may look for the minimum vibration amplitude to find the smallest possible dimensions. 


\section{Conclusions}

Engineers should customize the energy scavengers of the structural health monitoring systems by considering the ambient vibrations because the generated electricity is very high at the resonance frequency of these devices. Fitting them into the limited spaces of hard-to-reach locations and not to crack the piezoelectric element with overloading will be the other important concerns. The analytical models will not work very well since many assumptions will be required to model the the piezoelectric elements with very thin multiple layers. Also, the semirigid supports holding the piezoelectric beams will degrade the accuracy of the analytical models. FEM models take a very long time and require an optimization platform such as the GONNS. The feasibility of the use of the GONNS is evaluated in this paper, to design the optimal energy scavengers purely from the experimental data without considering any empirical equations or analytical models.

A simpler analytical model may also work well for optimization of the energy scavenger design if three important ingredients are correctly obtained: parameters, proper assumptions, and derivation.

a) First, the characteristic parameters of the beam should be obtained. Unless the correct parameters are provided by the manufacturer it is not easy to determine them experimentally. If the energy scavenger is going to operate at high temperatures the typically distributed parameters obtained at ambient conditions may need significant correction.

b) The boundary conditions of real applications may not be represented well by the analytical models. There is some flexibility in the clamping mechanism which holds the beam. This flexibility varies with the length of the beam and the mass. The mass has a significant contact area with the piezoelectric element instead of being held at a single line at the end of the beam. The strain is significantly different within this contact area. Depending on the length of the piezoelectric element and mass size, the model may need significant corrections.

c) It is not easy to derive and simplify the analytical model of a bimorph piezoelectric beam for estimation of the vibration amplitude and voltage generation at any given external excitation (amplitude and frequency). The bimorph material we used had seven layers on it. It is difficult to include the concerns in (a) and (b) in the model.

GONNS used either 2 or 3 input ANNs to represent the relationship between the inputs and outputs. Two different modes of the GONNS were used in the study. These approaches enabled the optimization either by using two 3-input ANNs with one GA or the three clusters each having two ANNs and one GA. The second approach is more reliable; however, it may recommend only one of the three masses used in the experiments. The training of the ANNs was a challenge because there were sharp spikes at the resonance frequencies of the beams, and working with multiple inputs made the problem worse. The estimation accuracy of the GONNS was improved using multipleclusters.

The study indicated that GONNS is a perfect platform to represent the experimental data in a very compact form using ANNs and to determine the optimal working conditions to maximize or minimize the concerned output parameter. 


\section{Acknowledgements}

This work was partially supported by the Florida International University, the Scientific and Technical Research Council of the Turkish Republic, and Istanbul Technical University.

\section{References}

1 A. Yenilmez, A. Yapici, C. Velez, I. N. Tansel and K. V. Jata: 33rd Annual Review of Progress in Quantitative Nondestructive Evaluation (AIP Conference Proceedings, Portland, 2007).

2 A. Yenilmez, A. Yapici, C. Velez and I. N. Tansel: 33rd Annual Review of Progress in Quantitative Nondestructive Evaluation (AIP Conference Proceedings, Portland, 2007) p. 902.

3 K. Zhang, Z. Han and G. Yu: Qing. Dax. Xue./J. Tsinghua University 39 (1999) 27.

4 R. Saravanan, P. Asokan and M. Sachidanandam: Int. J. Mach. Tools Manuf. 42 (2002) 1327.

5 T. Li, Y. H. Chen, F. Y. C. Boey and J. Ma: Sens. Actuators, A 134 (2007) 544.

6 A. J. Moskalik and D. Brei: Smart Mater. Struct. 8 (1999) 531.

7 S. Dong, X. H. Du, P. Bouchilloux and K. Uchino: J. Electron. 8 (2002) 155.

8 H. B. Fang, J.-Q. Liu, Z.-Y. Xu, L. Dong, L. Wang, D. Chen, B.-C. Cai and Y. Liu: Microelectron. J. 37 (2006) 1280.

9 R. Pérez, N. Chaillet, K. Domanski, P. Janus and P. Grabiec: Sens. Actuators, A 128 (2006) 367.

10 J. Kim and J. H. Lee: Smart Mater. Struct. 14 (2005) 934.

11 F. Lu, H. P. Lee and S. P. Lim: Smart Mater. Struct. 13 (2004) 57.

12 D. E. Rumelhart, G. Hilton and R. J. Williams: Computational Models of Cognition and Perception Series (MIT Press, Cambridge, 1986).

13 B. Widrow: DARPA Neural Network Study (AFCEA International Press, Fairfax, 1988).

14 L. Yan and K. Krishnamurthy: Dynamic Systems and Control Conference DSC (ASME, 2002) p. 105.

15 F. Cao and Q. Zhang: J. Mater. Process. Technol. 149 (2004) 106.

16 S. Y. Yang, V. Girivasan, N. R. Singh, I. N. Tansel and C. V. Kropas-Hughes: Int. J. Mach. Tools Manuf. 43 (2003) 175.

17 D. E. Goldberg: Optimization and Machine Learning (Addison-Wesley, Reading, 1989).

18 S. R. Ladd: Genetic Algorithms in C++ (M\&T Books, New York, 1996).

19 H. A. Sodano, D. J. Inman and G. Park: Strain 40 (2004) 49. 